\title{
Roadmap Development For Cloud Technology Implementation In Animation Industry (A Case Study)
}

\author{
Andrew Willis ${ }^{1}$, Prio Utomo ${ }^{2}$, P.M. Winarno ${ }^{3}$ \\ \{ andrew.willis@umn.ac.id ${ }^{1}$, prio.utomo@umn.ac.id ${ }^{2}$, pmwinarno@umn.ac.id $\left.{ }^{3}\right\}$
}

Magister Manajemen Teknologi, Universitas Multimedia Nusantara, Tangerang, Indonesia ${ }^{1}$,

Magister Manajemen Teknologi, Universitas Multimedia Nusantara, Tangerang, Indonesia ${ }^{2}$, Magister Manajemen Teknologi, Universitas Multimedia Nusantara, Tangerang, Indonesia ${ }^{3}$

\begin{abstract}
Animation industry in Asia is emerging with the growing demand of animation content following the ever increasing adoption of new OTT channel. Following the growth, animation studio has continuously seek a way to increase their competitive advantage by leveraging lower labor cost, opening satelite studio, new technology, or outsourcing production method. However, these CAPEX solution provide limited flexibility for the studio to adapt to various project specification which reduce production efficiency. Therefore, Cloud technology which boast OPEX solution is a novelties which offer not only efficiency but flexibility to animation production. This paper will follow a case study of an animation studio from company strategic analysis, roadmap development using CMM (Cloud Maturity Model) framework, and business case comparison with TCO (Total Cost of Ownership). The finding not only see the potential benefit of cloud implementation in animation industry but open up new business model possibility for the industry as well.
\end{abstract}

Keywords: Animation Industry, Cloud Technology, Digital Transformation, Cloud Maturity Model

\section{Introduction}

Animation industry in Asia is growing following the increase of digital media content demand which can be attributed by the growing subscriber of OTT (Over The Top) channel such as netflix, Hulu, and Hooq which able to provide digital content faster and more affordable as can be seen on the figure below. This phenomena has pushed the existing animation studio to ramp up the production and outsource their production to Asian countries which offer lower labor cost such as China, India, Singapore, and Phillipines while some other Asian countries follow such as Malaysia, Thailand, and Vietnam [1]. The boom of animation industry in Asia has prompted quick growth of new studio across the area offering ther services to the industry not only leveraging lower labor cost but also compete in scalability, technology, innovation, flexibility, and efficiency of their production process. This development process is endless and the industry keep looking for new ways to increase their competitive advantage among the industry player.

One form of technology that can answer the challenges faced by animation industry is cloud computing technology. Cloud computing is an ubiquitous model of on-demand computing 
resources which can be easily provisioned and deployed with minimal management effort [2]. Cloud computing technology has long become the playground for server application and basic desktop computing. However, with the introduction of PcoIP (PC Over Inter Protocol) VDI (Virtual Desktop Infrastructure) developed by Teradici has enabled animation industry to leverage the OPEX (Operational Expenditure) solution of cloud computing.

Implementing cloud is considered an unknown territory for most organization as there is no specific guidelines on how to implement cloud technology to the company business process especially with different business has different requirement which much often make cloud technology becomes an unknown territory for each case. Therefore, in this paper we will follow Cloud Maturity Model (CMM) framework released by OACA (Open Alliance for Cloud Adoption). CMM has been developed to help organisation to implement cloud technology platform into their business by looking at non-technical and technical component [3]. The framework will help us look at the company goals and strategy then develop a tools to assess current and future maturity stage within CMM framework which will help in developing a roadmap to cloud implementation.

The goal of this paper is to generate a roadmap within a case study in one of Asia animation studio to implement cloud technology which enable studio production process to be flexible to various incoming project, increase production efficiency, and develop potentially new animation studio business model using platform.

\section{Literature Review}

Cloud computing technology is seen as a way to provision a computing resources through a network with on-demand model and minimum management effort [2]. This technology enable company to not only move from CAPEX (Capital Expediture) solution to OPEX but also open up new business model opportunities which are convenient, flexible, and efficient.

VDI (Virtual Desktop Infrastructure) is one of cloud computing technology which enable virtualized desktop computer within centralized server environment. These virtualized desktop computer can be accessed through a remote terminal using various devices such as thin client, tablet, smartphone, PC, and laptop. These terminal will become a representation of the said desktop computer and will seems like a desktop computer exist within the terminal where in fact the entire processing happens in the centralized server [4]. One of emerging technology in VDI is called PcoIP developed by Teradici which generate a stream of image data of the virtualized desktop and send them to terminal using encryption key whereas the classic RDP (Remote Desktop Protocol) will send the entire I/O instead.

\begin{tabular}{|c|c|c|c|c|c|}
\hline$\underset{\text { Legacy }}{\text { CMM } 0}$ & $\begin{array}{l}\text { CMM } 1 \\
\text { Inifial, Ad hoc }\end{array}$ & $\begin{array}{c}\text { CMM } 2 \\
\text { Repeatable, 0pportunistic }\end{array}$ & $\begin{array}{c}\text { CMM } 3 \\
\text { Defined, Systematic }\end{array}$ & $\begin{array}{c}\text { CMM } 4 \\
\text { Measured, Measurable }\end{array}$ & $\begin{array}{l}\text { CMM } 5 \\
\text { Optimized }\end{array}$ \\
\hline
\end{tabular}

Fig. 1. CMM Maturity Stage

CMM (Cloud Maturity Model) is a cloud adoption frameworks developed by OACA (Open Alliance for Cloud Adoption) which often used to help an organization to implement cloud technology within their business process from technical or non-technical component. The framework consist of 6 maturity stage as seen in fig 1 to help clasify company cloud maturity stage. The framework take into account company business goals and strategy which enable the 
user to generate a set of questionnaire to measure their company maturity stage and develop an implementation roadmap from it [3]. There are two capabilities that can be used in CMM, the technical and non-technical capabilities. Technical capabilities focus more on technical side of cloud computing technology while non-technical focus on management side of cloud computing implementation, each with their own set of domain. In this case, we will use non-technical capability as seen on fig. 2 .

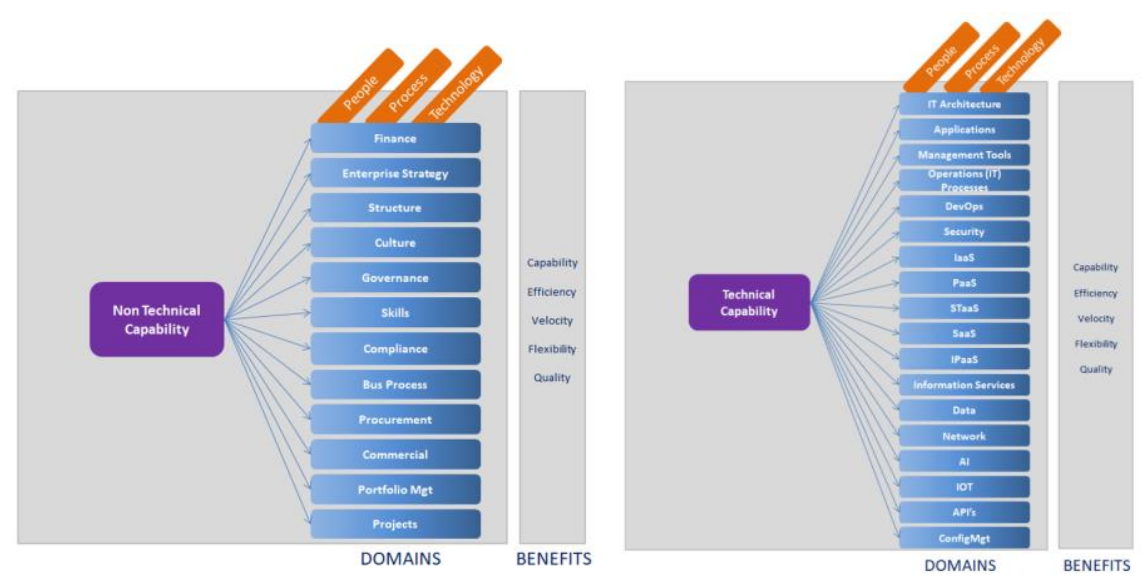

Fig. 2. CMM Capabilities

\section{Methodology and Data Analysis}

In order to be able to generate a roadmap, CMM maturity assessment tools frameworks will be used. This tools will be in form of questionnaire that can be taylored to align with company business goals. In this part we will see the forming of CMM questionnaire for the animation studio case.

First step is to align the CMM frameworks with the studio business goals. This can be done by reviewing the company strategic document or in animation studio case through vision/mission statement review and initial interview with stakeholders. This business goals will then reviewed to analyze common responses taken by the industry then against a list of CMM use case. This will reveal the company targeted maturity in CMM framework while keep aligned with company business goals as well as the time require to reach the future maturity stage.

Next, the identified use case can be analyzed against selected domains capabilities (technical or non-technical capabilities). Not all domain will be selected as some domain might be more relevant for a company while other might not relevant to company business model. In the animation studio case a total of 7 domains deemed relevant based on company business model and industry condition.

Once the domain has been identified, the questionnaire can be generated from list of questionnaire bank within the frameworks. All questionnaire fall into 3 groups for each domain which is people, process, and technology. Each questionnaire is a micro indicator for each domain and contains brief description for each maturity stage. The completed questionnaire will go through validity and reliability due to the questionnaire required to be translated to local language. 
The finished questionnaire then used to assess the company (animation studio) current Cloud Maturity stage with the company managers. During the interview the company business goals along with maturity stage goals will also be confirmed with the manager. The questionnaire in the case study consists of 39 variables for 7 different domains relevant to the animation studio case.

The maturity stage data collected from the interview will then be averaged for each domain and can be used for gap analysis through spider chart shown above which in the animation studio case is considerably large due to no previous cloud technology implementation ever attempted before. Next, from each of the maturity parameter data from the interview, we can identify action plan for each maturity stage from current stages to future stages. These action plan ultimately criss-crossing one domain to another where some action plan can be grouped into one big program or eventually combined into one same activity. The roadmap then further simplified by classified into each sub-layer (people, process, and technology)

\section{Research Result and Discussion}

Final goal of this research is to develop a roadmap for cloud technology implementation in one of Asia animation studio. This part will discuss the questionnaire result of the said case, its gap analysis within the CMM maturity stages, and final roadmap disucssion.

Table 1. Questionnaire Result
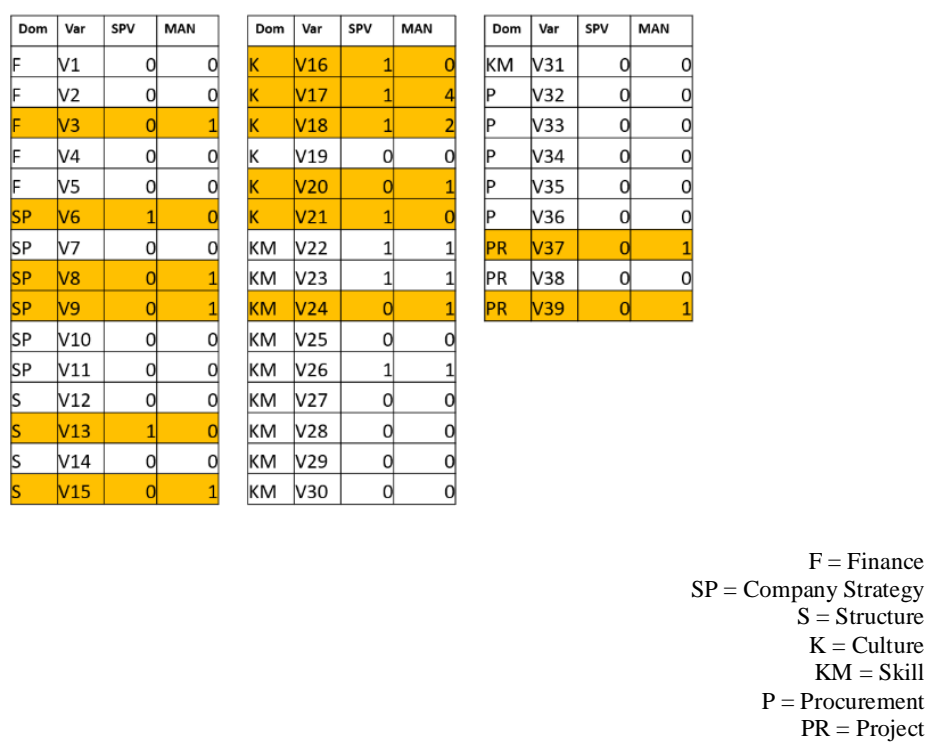

The questionnaire result shown above show the current cloud maturity stage according to the company supervisors and the managers. Note that on certain domains, the supervisor and manager has more different point of view on current stage mainly in culture $(\mathrm{k})$, compange stragey (sp), and structure (s) with highest difference can be seen in culture (k) on variable 17. The author believe that this is due to the nature of the questions that solely exclusive to 
stakeholders or decision maker domain and thus certain strategic information is unfamiliar to supervisors.

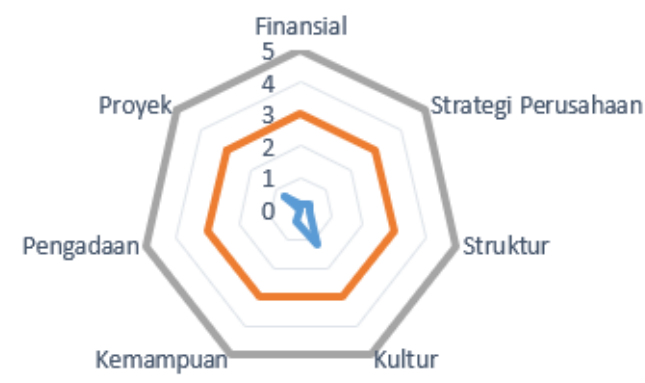

Fig. 3. Maturity gap spider chart

The gap analysis can be seen on the gap chart above. Note that almost all domain has large gap between current maturity stage and the future stage. This is due to the company in the case (animation studio) has not prior cloud technology implementation effort and thus various aspect of the cloud itself has been absent from the company. Only one domain that seems to be more advance than other which is the culture. This is due to animation company tend to have open minded nature towards innovation which provide basic maturity for cloud implementation.

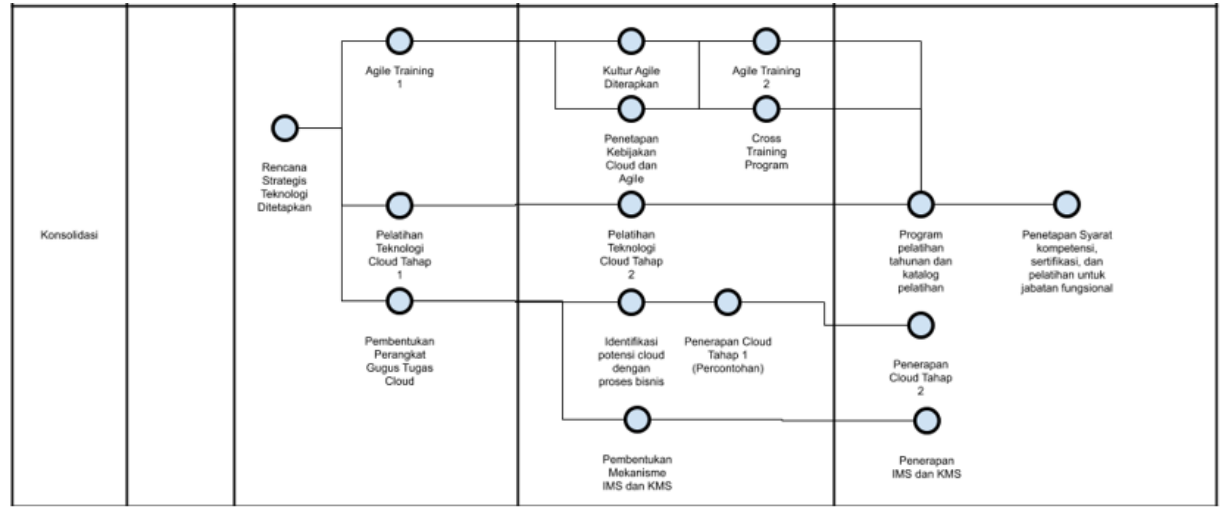

Fig. 4. People perspective roadmap

The roadmap for people perspective in the first stage focus on planning, basic training (for both cloud and new culture supporting the cloud technology), and forming a new task force for cloud implementation. The second stage will focus on advance training, transitioning incumbent business process to cloud mechanism, and institutionalized innovation and knowledge within the studio. The third stage focus on implementing standardized system in manpower and continuous training for each company structural function. 


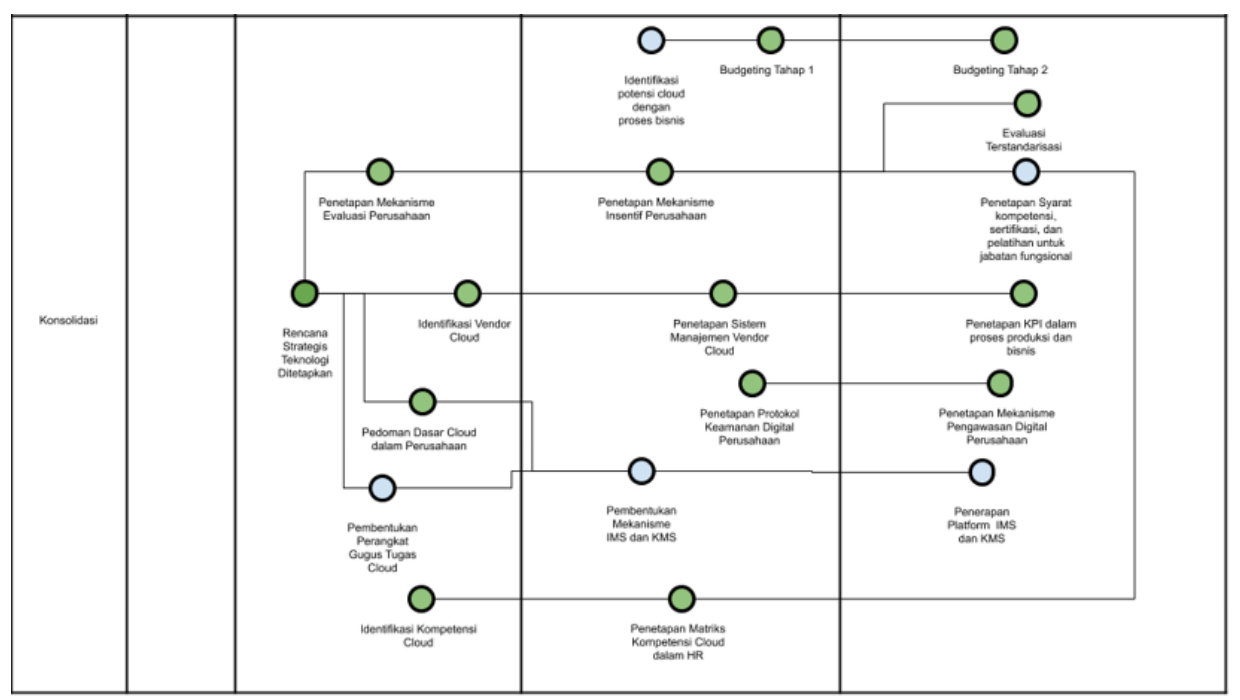

Fig. 5. Process perspective roadmap

In the first stage for process perspective focus on identifying and scouting compatibility with the new cloud technology with certain areas criss-cross with people persepective. The second stage focus on instituionalized the cloud system and other company process mechanism such as incentive and security protocol. The last stage consist of implementation and auditing.

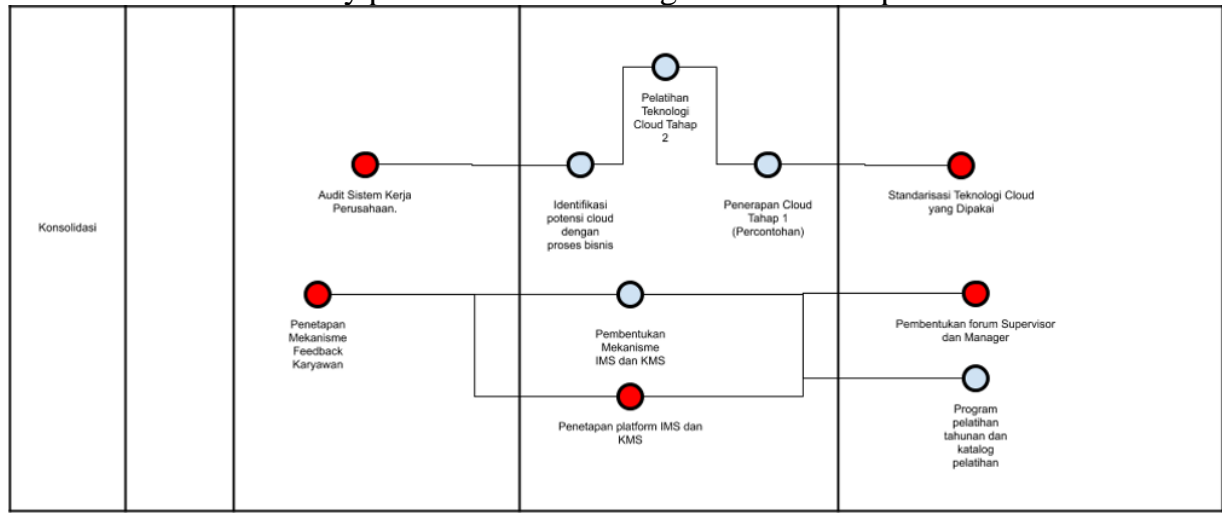

Fig. 6. Technology perspective roadmap

The technical perspective is relatively simple as majority of the process has been mentioned on other perspective. The first stage focus on technology self assessment while second stage and final stage focus more on gradual technology implementation and continuous improvement program.

Each of the point in the roadmap represent a group of action developed based on the framework following the company business goals. Each action can then be elaborated more during implementation. 


\section{Conclusion}

There is no certain standards in implementing a novel technologies. In this paper, CMM has shown that it can be utilized to help develop a roadmap for cloud technology implementation by one of Asia animation studio. The framework has a method to align itself with the company business goals and therefore able to taylor a customized questionnaire which able to measure current maturity stage of the company. Using the gap analysis, the company using CMM customized questionnaire structure can generate a rodmap to implement and harness the potential of cloud computing technology.

\section{Implications / Limitations And Suggestions For Further Research}

Every business has its own environment and limitation. Therefore, it is imperative that in implementing CMM framework that one has to consider each relevancy of the domain, use case, and questionnaire bank to its case. Animation industry which begin to gain momentum in implementing cloud technology might seem difficult to implement CMM framework due to few cloud computing implementiation and use case in animation industry.

Further study in developing a clear, concise, and more systematic relevancy between CMM current framework and animation industry use case is needed in the future. Notable areas to be studied further included but not limited to developing use case bank for animation industry in CMM framework, method development in defining domain relevancy, and roadmap development. This future improvement will enhance CMM reliability and accuracy in determine animation studio maturity stage and plan their cloud technology adoption.

\section{References}

[1] H. S. Kamarudin and K. Sajilan, "Critical Success Factors of Technopreneurship in the Creative Industries : A Study of Animation Ventures," Rev. Integr. Bus. Econ. Res., vol. 2, no. 1, pp. 137,2013

[2] P. Mell and T. Grance, "The NIST-National Institute of Standars and Technology- Definition of Cloud Computing,” NIST Spec. Publ. 800-145, p. 7, 2011.

[3] M. Williams, R. Skipp, T. Scott, and M. Estes, "OACA Cloud Maturity Model v4.0," no. June, pp. 0-62, 2018.

[4] M. Rouse, "What is Virtual Desktop Infrastructure (VDI) and How Does it Work?," 2020. [Online]. Available: https://searchvirtualdesktop.techtarget.com/definition/virtual-desktopinfrastructure-VDI. [Accessed: 30-Jun-2020]. 